\title{
CORRELATION OF MODE OF INJURY, CLINICAL PRESENTATION TO THE ORGAN INJURY AND MANAGEMENT IN ABDOMINAL TRAUMA
}

\author{
Naga Aparna Peri ${ }^{1}$, Rahul R. Bhat², Poornachandra Thejeswi D. S3, B. K. Shivaprasad Rai ${ }^{4}$
}

1Junior Resident, Department of General Surgery, Kasturba Medical College, Mangalore, Karnataka. ${ }^{2}$ Associate Professor, Department of General Surgery, Kasturba Medical College, Mangalore, Karnataka. ${ }^{3}$ Associate Professor, Department of General Surgery, Kasturba Medical College, Mangalore, Karnataka. 4 Professor, Department of General Surgery, Kasturba Medical College, Mangalore, Karnataka. \begin{abstract}
BACKGROUND

Trauma is still the most frequent cause of death in the first four decades of life and it remains a major public health problem in every country. The abdomen is one of the most commonly injured region requiring surgical intervention in about $25 \%$ of cases. Abdominal trauma is traditionally classified as either blunt or penetrating. The peak incidence of abdominal trauma occurs in second to third decade with majority of patients being males.

Aim- This prospective, observational, longitudinal study aims to correlate the modes of abdominal injury with its presentation (organ injured in trauma) and the management of the same.
\end{abstract}

\section{MATERIALS AND METHODS}

The study was conducted in Kasturba Medical College, Mangalore for the period of one and a half years. All the patients aged between 18-65 years with blunt or penetrating abdominal trauma were included in the study. Patients with head injury, thoracic injury with connective tissue diseases on corticosteroids, patients with abdominal TB, patients with inflammatory bowel disease, post splenectomy, post cholecystectomy were excluded from the study. The results were analysed using Chi-square test.

\section{RESULTS}

A total of 106 patients with blunt and penetrating abdominal trauma were observed during the period of study. Various modalities of blunt and penetrating injuries of the abdomen and the organs injured in each modality were observed. All penetrating abdominal injuries were operated, whereas most of the blunt injury abdomen cases were managed conservatively.

\section{CONCLUSION}

Through this study, it can be concluded that different modalities of abdominal trauma have different presentations with respect to organs involved.

\section{KEYWORDS}

Trauma, Blunt Trauma Abdomen, Penetrating Trauma Abdomen, Conservative Management.

HOW TO CITE THIS ARTICLE: Peri NA, Bhat RR, Thejeswi PDS, et al. Correlation of mode of injury, clinical presentation to the organ injury and management in abdominal trauma. J. Evolution Med. Dent. Sci. 2018;7(13):1611-1615, DOI: $10.14260 /$ jemds/2018/365

\section{BACKGROUND}

Injuries are an important public health concern and remains a growing problem in some countries. Two of the three leading causes of injury deaths- road traffic injuries and falls are predicted to rise in rank compared to other causes of death.(1) Road traffic injuries are predicted to become the 7th leading cause of death by 2030 with falls rising to become the $17^{\text {th }}$ leading cause of death and suicide remaining in the top 20. (1) The annual contribution of injury related mortality is estimated around 5 million, which is approximately $9 \%$ of the total deaths occurring in the world. This is nearly 1.7 times the number of fatalities that result from HIV/ AIDS, tuberculosis and malaria combined. Approximately, a quarter of the 5 million deaths from injuries are the result of suicide

'Financial or Other Competing Interest': None.

Submission 12-02-2018, Peer Review 07-03-2018,

Acceptance 14-03-2018, Published 26-03-2018.

Corresponding Author:

Dr. Naga Aparna Peri,

Flat No. C-5, Clove Gardens,

New Balmatta Road, Falnir,

Mangalore-575001, Karnataka.

E-mail:aparna116@gmail.com

DOI: $10.14260 /$ jemds $/ 2018 / 365$

\section{(c) (i) $(3)$}

and homicide, while road traffic injuries account for nearly another quarter. Other main causes of death from injuries are falls, drowning, burns, poisoning and war.

Injury related deaths are largely preventable through proper application of safety measures and other primordial and primary preventive measures. Collaboration between various organisations, governments, law and order agencies might decrease the burden of injury related deaths.(1) Abdominal trauma is classified as either blunt or penetrating.

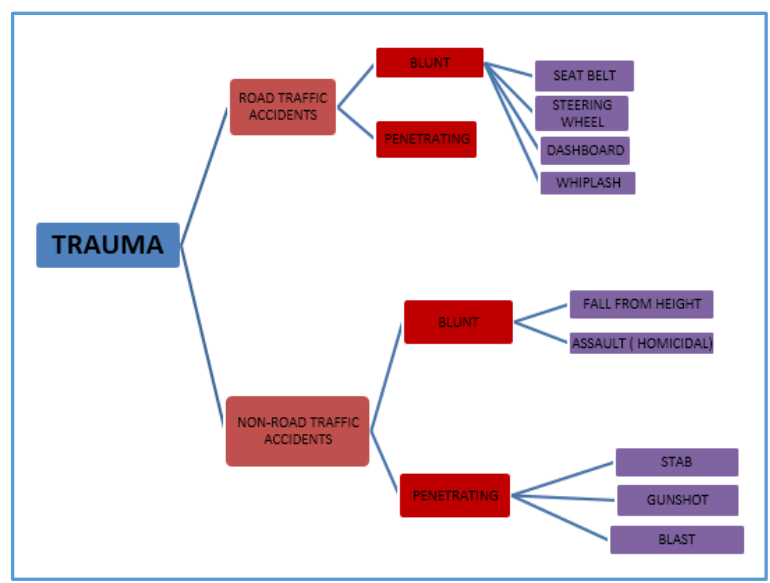

Figure 1. Classification of Trauma 


\section{Blunt Trauma Abdomen}

Blunt abdominal trauma is a leading cause of morbidity and mortality among all age groups. Identification is often challenging, as there can be internal haemorrhage, multiple injuries and delayed manifestation. Many injuries may not manifest during the initial assessment and treatment period. Around $2 / 3^{\text {rd }}$ of blunt injuries of abdomen are related to vehicular trauma. Patterns of organ involvement variable for various mechanisms of injury. Nowadays, most of the blunt abdominal injuries are managed conservatively.

\section{Penetrating Trauma Abdomen}

Penetrating trauma abdomen involves the violation of the abdominal cavity. Its clinical presentation depends on various factors such as type of penetrating weapon or object, the range of occurrence of injury, organs involved and location and number of wounds. The management of penetrating trauma has evolved from conventional surgical management towards conservative management in stable cases with involvement of interventional radiology.

\section{Aims and Objectives}

This study aims to compare the mode of injury to clinical presentation and organ injured in abdominal trauma and also to compare the outcome of the injury, i.e. surgical or conservative management.

\section{MATERIALS AND METHODS}

This is a prospective observational study that was conducted in Kasturba Medical College, Mangalore for a period of one and a half years. A total of 106 patients were included in the study. After admission to the Emergency Department, a detailed history regarding the mode of injury was taken and physical examination was done. All appropriate laboratory investigations- Haemoglobin, Total Leukocyte Count, Differential Leukocyte Count, Packed Cell Volume, Random Blood Sugar, Serum Electrolytes, Renal Function Tests, Liver Function Tests, HIV and HBsAg were sent. Radiological investigations included Chest $\mathrm{x}$-ray, Erect x-ray abdomen, USG, CECT abdomen and pelvis +/- Cystogram. Patients between 18 - 65 years with blunt or penetrating abdominal trauma were included in this study. The patients who were excluded from the study were:

- Patients with head injury.

- Thoracic injury.

- With connective tissue diseases on corticosteroids.

- Patients with abdominal TB.

- Inflammatory bowel disease.

- Post splenectomy, post cholecystectomy.

Depending upon the organs involved and the haemodynamic stability, patients were either managed conservatively or surgically. All the data were analysed using a Chi-square test using the SPSS - 20 software. A ' $p$ ' value of $<0.05$ was considered significant.
RESULTS

Age and Sex Distribution

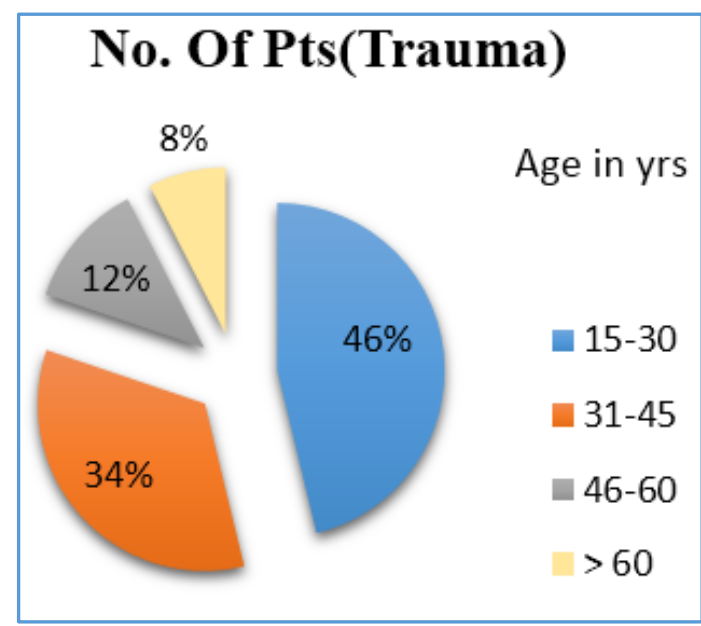

Figure 2

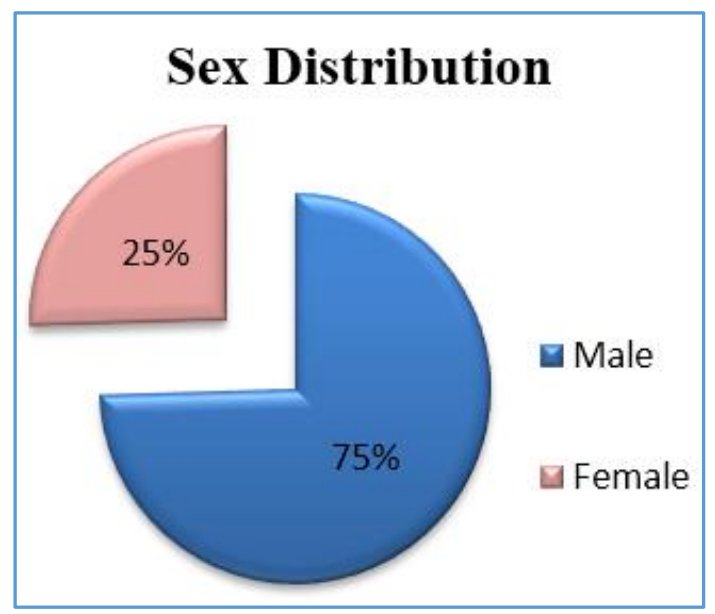

Figure 3

\section{Age and Sex Distribution in Trauma Victims}

Of the 106 patients in this study 85 patients sustained blunt trauma, while 21 patients sustained penetrating trauma. Most of the patients were in the age group of $15-30$ yrs. followed by $31-45$ yrs. Males were the predominant gender involved.

\section{Modes of Injury}

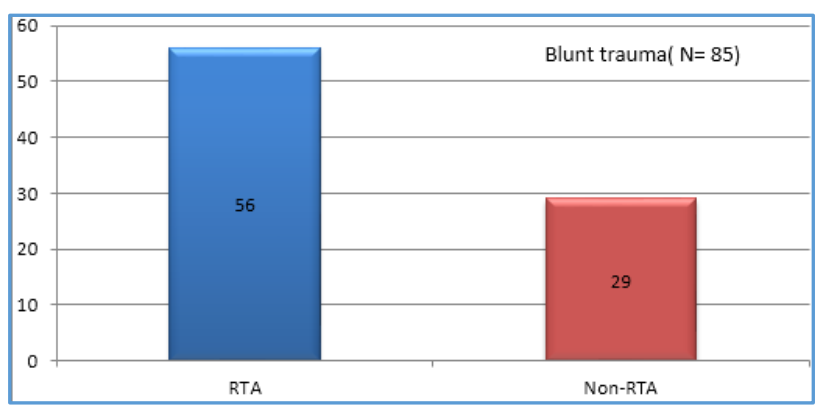

Figure 4 


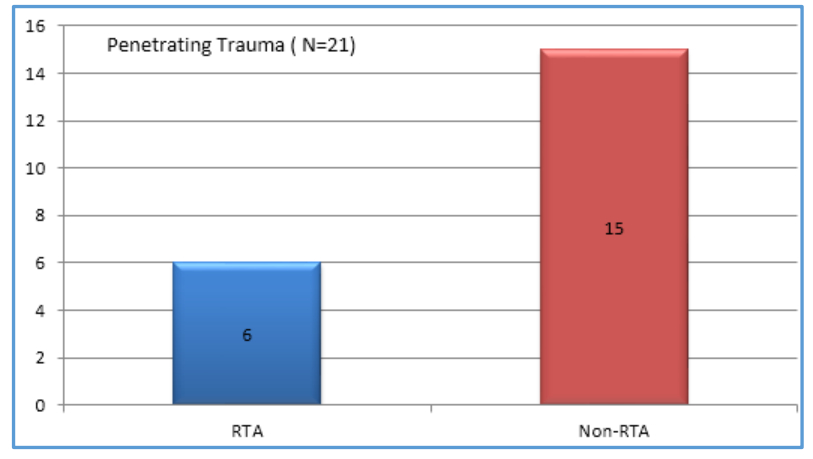

Figure 5

In this study 85 patients sustained blunt trauma, while 21 patients sustained penetrating trauma. While comparing the modes of injury most of the abdominal trauma was caused due to road traffic accidents, while this trend was reversed in penetrating trauma wherein most of the injuries were due to non-RTA aetiology $(\mathrm{p}=$ 0.003).

\begin{tabular}{|c|c|c|c|}
\hline \multirow{2}{*}{$\begin{array}{c}\text { Mechanism } \\
\text { of Injury }\end{array}$} & Blunt & Penetrating & \multirow{2}{*}{ P value } \\
\cline { 2 - 3 } & 56 & 6 & \multirow{2}{*}{0.003} \\
\hline RTA & 29 & 15 & \\
\hline Non-RTA & \multicolumn{3}{|c|}{ Table 1 } \\
\hline
\end{tabular}

Patterns of Organ Injury (Blunt Trauma)

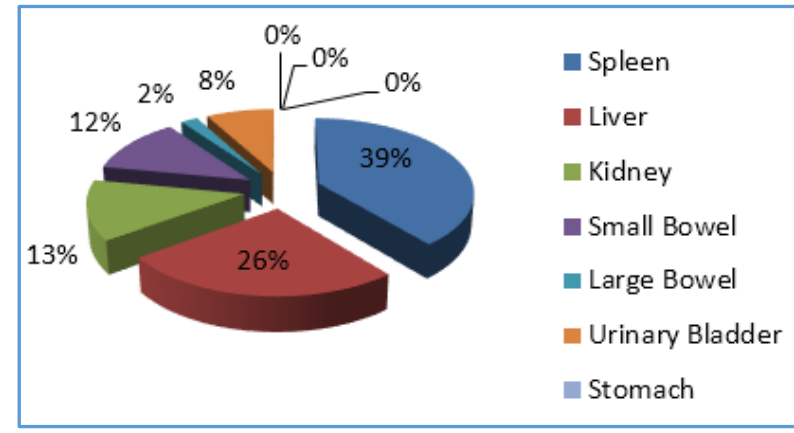

Figure 6

\begin{tabular}{|c|c|c|}
\hline Organ Involved & RTA & NON - RTA \\
\hline Spleen $(\mathrm{n}=39)$ & 25 & 14 \\
\hline Liver $(\mathrm{n}=26)$ & 19 & 7 \\
\hline Kidney $(\mathrm{n}=13)$ & 7 & 6 \\
\hline Small Bowel $(\mathrm{n}=12)$ & 8 & 4 \\
\hline Large Bowel $(\mathrm{n}=2)$ & 1 & 1 \\
\hline Urinary Bladder $(\mathrm{n}=8)$ & 5 & 3 \\
\hline Stomach & 0 & 0 \\
\hline Pancreas & 0 & 0 \\
\hline Gall Bladder & 0 & 0 \\
\hline
\end{tabular}

Amongst the organs injured in blunt trauma abdomen, spleen was the most common organ involved followed by liver and kidney. Among the hollow viscera, small bowel was most commonly involved. There were no gall bladder or pancreatic injuries observed. While comparing the organ involvement in RTA and non-RTA, spleen was the most common organ injured in RTA while kidney was the most common solid organ injured in non-RTA.

\section{Patterns of Organ Injury (Penetrating Trauma)}

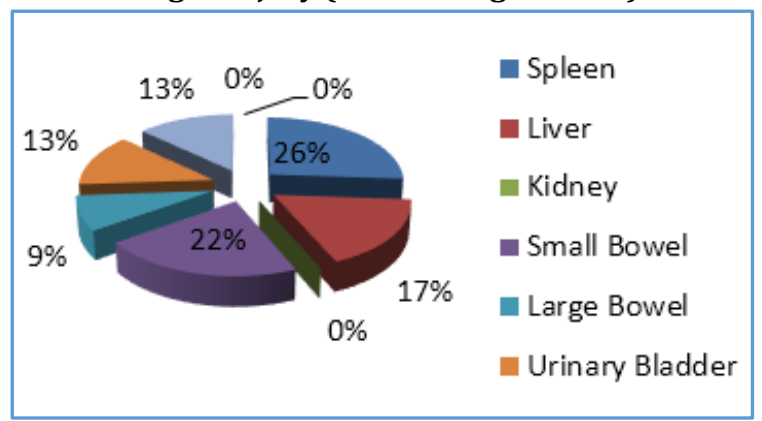

Figure 7

\begin{tabular}{|c|c|c|}
\hline Organ Involved & RTA & Non-RTA \\
\hline Spleen $(\mathrm{n}=6)$ & 1 & 5 \\
\hline Liver $(\mathrm{n}=4)$ & 2 & 2 \\
\hline Kidney $(\mathrm{n}=0)$ & 0 & 0 \\
\hline Small Bowel $(\mathrm{n}=5)$ & 0 & 5 \\
\hline Large Bowel $(\mathrm{n}=2)$ & 0 & 2 \\
\hline Urinary Bladder(n = 3) & 1 & 2 \\
\hline Stomach (n = 3) & 1 & 2 \\
\hline Pancreas & 0 & 0 \\
\hline Gall Bladder & 0 & 0 \\
\hline
\end{tabular}

Table 3. Distribution of Organ Involvement in Penetrating Trauma

In penetrating trauma abdomen, spleen and small bowel injury predominated followed by liver, urinary bladder and stomach.

Time of Presentation/Blunt Trauma

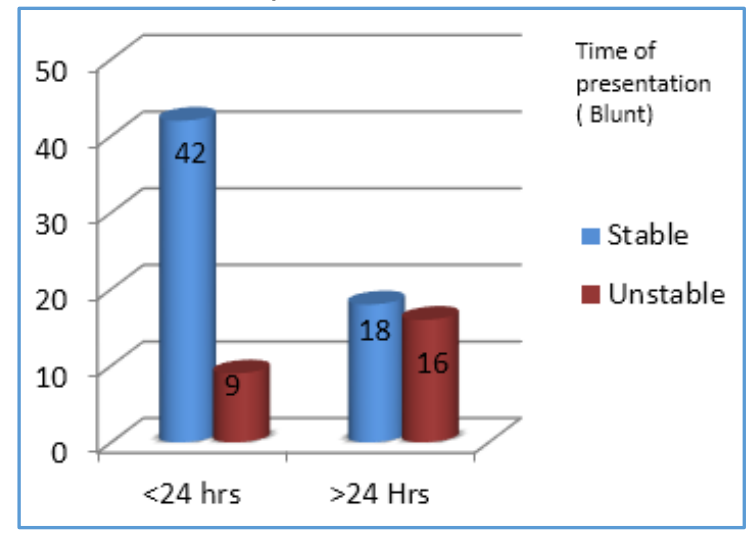

Figure 8

Penetrating Trauma

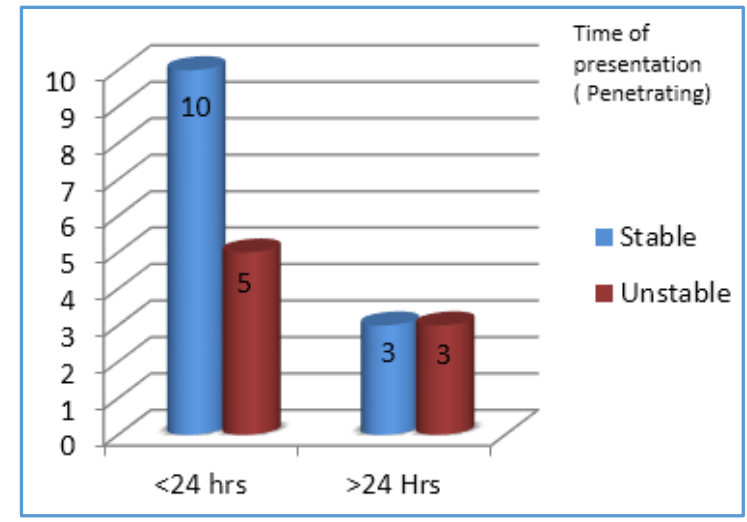

Figure 9 
Patients who presented to the hospital within $24 \mathrm{hrs}$. of injury had a favourable outcome with respect to haemodynamic stability and mortality compared to those who presented after 24 hrs. of injury ( $p=0.006)$.

\begin{tabular}{|c|c|c|c|}
\hline Time of & Haemodynamic Stability & \multirow{2}{*}{ P value } \\
\cline { 2 - 3 } Presentation & Stable & Unstable & \\
\hline Less than 24 hrs. & 52 & 14 & 0.006 \\
\hline More than 24 hrs. & 21 & 19 & \\
\hline \multicolumn{3}{|c|}{ Table 4 } \\
\hline
\end{tabular}

\section{Management \\ Blunt Trauma}

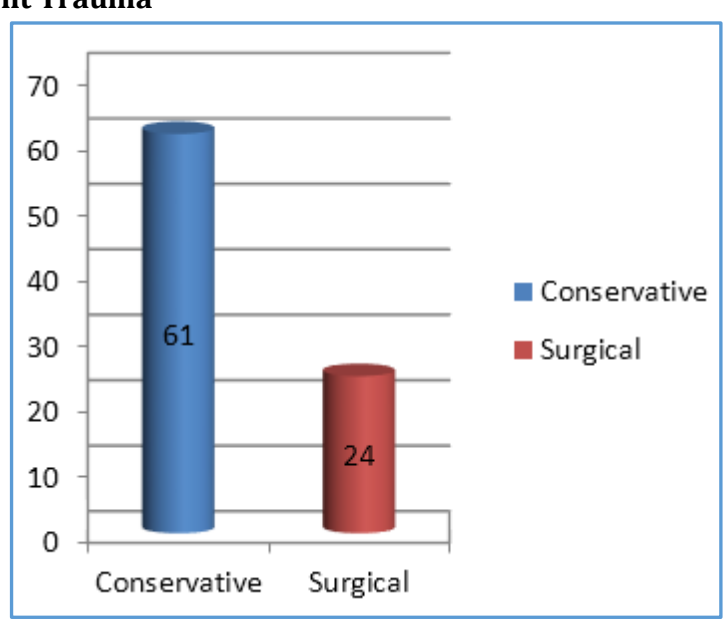

Figure 10

Penetrating Trauma

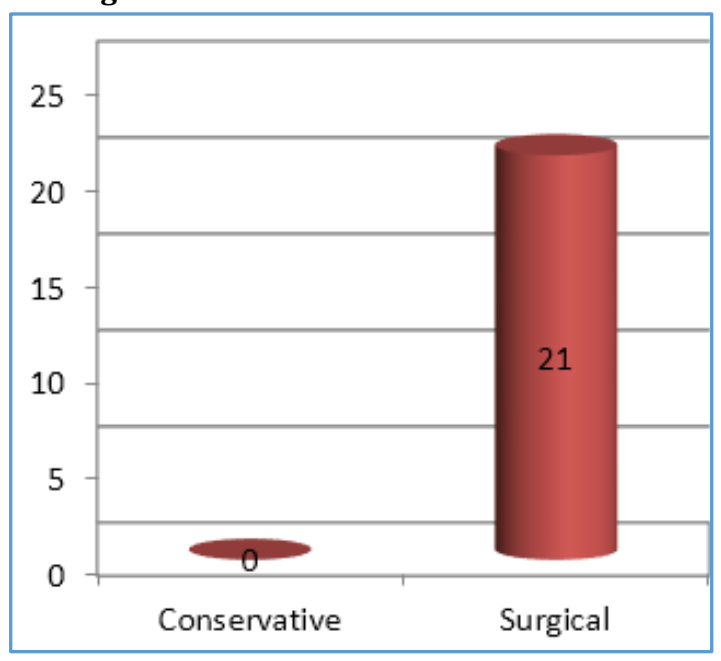

Figure 11

Majority of the patients with blunt trauma abdomen were managed conservatively. However, some patients who were haemodynamically unstable were managed surgically. Hollow viscus injuries due to blunt trauma were managed surgically. All the penetrating trauma cases, which presented to the ER were managed surgically.

\begin{tabular}{|c|c|c|c|}
\hline \multirow{2}{*}{ Type of Trauma } & \multicolumn{2}{|c|}{ Management } & \multirow{2}{*}{ P value } \\
\cline { 2 - 3 } & Conservative & Surgical & \multirow{2}{*}{$<0.001$} \\
\hline Blunt Trauma & 61 & 24 & 21 \\
\hline Penetrating Trauma & 0 & Table 5 \\
\hline \multicolumn{3}{|c|}{} \\
\hline
\end{tabular}

\begin{tabular}{|c|c|c|c|}
\hline \multirow{2}{*}{ Management } & Haemodynamic Stability & \multirow{2}{*}{ P value } \\
\cline { 2 - 3 } & Stable & Unstable & \multirow{2}{*}{$<0.001$} \\
\hline Conservative & 52 & 9 & \\
\hline Surgical & 21 & 24 & \\
\hline \multicolumn{3}{|c|}{ Table 6 } \\
\hline
\end{tabular}

\section{DISCUSSION}

The present study was designed to correlate the mode of injury, clinical presentation to the organ injury and management in abdominal trauma. This study is the first of a kind in the district of Dakshina Kannada, Karnataka. It is hoped that through this study, the epidemiological aspects of trauma would be recognised and appropriate measures would be set in place to decrease the burden of trauma.

Out of a total of 106 patients enrolled into this study, most of them were falling between the age group of $15-30$ years $(n=49)$. The number of patients in the age group $31-45$ years was also significant $(n=36)$. Most of the patients were males $(n=79)$. This pattern can be attributed to the fact that males are more ambulant when it comes to going to work or carrying out any other activity. Hence, they have a higher chance to get exposed to the risk factors of trauma. These findings are in line with the global burden of injuries as per the WHO statistics (1) and many other studies. Nikhil Mehta et al from the Dept. of General Surgery, Kempegowda Institute of Medical Sciences, Bangalore also report a similar pattern of distribution in their experience on blunt trauma abdomen. (2) The contribution of automobile related injuries in this study was $58.5 \%(n=62)$, whereas non-RTA injuries were $41.5 \%$ $(n=44)$. Most of the injuries due to non-RTA causes were due to fall from height.

Blunt trauma abdomen constituted a major share of the total trauma cases seen at our institute $(n=85 ; 80.18 \%)$, out of which $66 \%(n=56)$ were due to RTA. This region is prone for road traffic accidents owing to narrow roads, ghat roads in the outskirts of Mangalore and erratic driving by the citizens. A similar pattern was observed by R Balamurugan et al,(3) D. Sah and S. P. Sinha, (4) Mohammad A Gad et al(5) in their study of blunt trauma abdomen.

While this is the pattern observed in blunt abdominal trauma, penetrating abdominal trauma $(n=21 ; 19.8 \%)$ was mainly due to non-RTA causes $(n=15)$. The most common modality was fall from height. Most commonly affected people were males, who were either labourers or construction workers who regularly scale certain heights during their work. The pattern of solid organ involvement in blunt abdominal trauma was more inclined towards spleen followed by liver. Kidney and small bowel were involved in an almost equal frequency followed by Urinary bladder involvement. It did not make any difference whether the mechanism of injury was RTA or non-RTA, as in both the mechanisms spleen was found to be the most common solid organ involved (Refer Table 2). In penetrating abdominal trauma, splenic injury and small bowel injury were found to be occurring equally and these were mostly due to non-RTA causes. Injuries of liver, stomach and urinary bladder followed (Refer Table 3).

It was surprising to note that there were no kidney injuries noted in penetrating trauma abdomen. Although D Sah et al(4) noted some kidney injuries due to penetrating trauma, most of the literature points towards the involvement of hollow viscus. Hence, the lack of kidney injury 
in penetrating trauma in the present is purely co-incidental. But we can think of certain factors that could have led to this observation such as the way the injury has occurred (e.g. angle of fall), the retroperitoneal position of the kidneys etc.

In both the modalities of injuries, patients who presented to the ER within 24 hours of the injury were haemodynamically stable. This observation has a strong statistical correlation $(p=0.006)$. The earlier the patient presents to the ED, the earlier the resuscitative measures can be started and this leads to a better patient outcome. Most of the patients with blunt trauma in our study presented within 24 hrs. (51/85; 60\%), out of which 42 were haemodynamically stable $(82.3 \%)$.

Most of the patients with penetrating trauma also presented within 24 hours and were haemodynamically stable (Ref. Fig. 9). About $50 \%$ of the patients who presented after $24 \mathrm{hrs}$. of blunt and penetrating trauma were haemodynamically unstable.

This could be because of the fact that some of them took a lot of time to reach our institute, and also some of them were transferred from other medical facilities. Owing to the inadequate resuscitative measures that could have happened during the transit, the haemodynamics of those patients were not good by the time they reached our institute. Solid organ injury of higher grades would lead to significant haemodynamic instability. Also, as the number of organs involved increases, the chances of haemodynamic instability also increases. Occurrence of pneumothorax, pericardial tamponade and haemorrhagic shock due to injury to the great vessels would lead to haemodynamic collapse, and attending to these issues should be of prime importance. That is why, these issues have to be addressed during the primary survey as per the ATLS protocol.

It is obvious that every minute lost in starting proper resuscitative measures leads to significant haemodynamic instability, poorer outcome and death. Also finally, as the age progresses the compensatory mechanisms of the circulatory system are deranged, hence making a geriatric individual more prone for haemodynamic instability.

Out of 33 haemodynamic unstable patients, 11 patients had multiorgan involvement. Most of the haemodynamically unstable patients had either splenic injury or liver injury of a higher grade (III - V) or both. This shows the importance of detecting solid organ injury early. There were 15 deaths among those who were haemodynamically unstable. The various factors for this poor outcome were those mentioned above. The most important being involvement of Spleen and Liver. There were 2 deaths in patients who were haemodynamically stable group, and this was probably due to massive pulmonary thromboembolism.

When it comes to the management of trauma cases, $57.5 \%$ of the blunt trauma cases were managed conservatively (61/106). Out of the remaining 24 cases, 16 blunt trauma cases were haemodynamically unstable and needed surgical exploration ( $p<0.001$ ). If there was involvement of hollow viscus, then surgical exploration was done. There were 9 casualties in those who were not surgically explored. These patients succumbed to the grievous injuries and any attempt for surgical intervention would have been futile. All the penetrating trauma cases were explored surgically as per the protocol of our institution. There is ample literature, wherein there is a shift towards a conservative approach in managing penetrating abdominal trauma involving solid organs and hollow viscus organs.(6),(7),(8) But any trauma involving the hollow viscus organs were managed surgically in this study.

\section{CONCLUSION}

In the present study, most of the trauma cases were that of blunt trauma. Most of the patients were in the age group of 15-45 years. Spleen was the commonly injured organ in blunt trauma followed by Liver and Kidney. Similar trend was observed in penetrating trauma also with significant involvement of small bowel.

Better clinical conditions and outcomes were noticed when patients presented to the ER early (within $24 \mathrm{hrs}$. of injury). Most of the blunt trauma cases were managed conservatively. Solid organ injuries in blunt trauma were managed conservatively. All hollow viscus injuries in blunt trauma were managed surgically. All the penetrating trauma cases were managed surgically.

\section{REFERENCES}

[1] World Health Organisation. Injuries and Violence: The facts 2014. Geneva: WHO, 2014.

[2] Mehta N, Babu S, Venugopal K. An experience with blunt abdominal trauma: evaluation, management and outcome. Clinics and Practice 2014;4(2):599.

[3] Balamurugan R, Kumar S, Lakshmana R, et al. Intraabdominal organ injuries in blunt injury abdomen in SRM Medical College, Potheri: a prospective study. IJSS Journal of Surgery 2016;2(2):37-42.

[4] Sah D, Sinha SP. Pattern of abdominal injuries in a tertiary care centre in Western UP. Annals of International Medical and Dental Research 2016;2(6):5-9.

[5] Gad MA, Saber A, Farrag S, et al. Incidence, patterns and factors predicting mortality of abdominal injuries in trauma patients. N Am J Med Sci 2012;4(3):129-34.

[6] Feliciano DV, Rozycki GS. The management of penetrating abdominal trauma. Adv Sur 1995;28:1-39.

[7] Zinner MJ, Ashley SW. Management of abdominal trauma. Chapter 12. Maingot's abdominal operations. $12^{\text {th }}$ edn. McGraw-Hill Professional 2012.

[8] Raza M, Abbas Y, Devi V, et al. Non operative management of abdominal trauma - a ten year review. World Journal of Emergency Surgery 2013;8:14. 